\title{
LARGE AUTOMORPHISM GROUPS OF COMPACT KLEIN SURFACES WITH BOUNDARY, I
}

\author{
by COY L. MAY
}

(Received 7 March, 1975; revised 18 November, 1975)

0. Introduction. Let $X$ be a Klein surface [1], that is, $X$ is a surface with boundary $\partial X$ together with a dianalytic structure on $X$. A homeomorphism $f: X \rightarrow X$ of $X$ onto itself that is dianalytic will be called an automorphism of $X$.

In a recent paper [8] we showed that a compact Klein surface of (algebraic) genus $g \geqq 2$, with non-empty boundary, cannot have more than $12(g-1)$ automorphisms. We also showed that the bound $12(g-1)$ is attained, by exhibiting some surfaces of low genus $(g=2,3,5)$ together with their automorphism groups. The corresponding bound for Riemann surfaces is quite well known; Hurwitz showed that a compact Riemann surface of genus $g \geqq 2$ has at most $84(g-1)$ (orientation-preserving) automorphisms.

Here we study those groups that act as a group of $12(g-1)$ automorphisms of a compact Klein surface with boundary of genus $g \geqq 2$. Our main result is a characterization of these groups in terms of their presentations. We call these finite groups $M^{*}$-groups. It is easy to find examples of $M^{*}$-groups. In fact, by using known results about normal subgroups of the modular group, we are able to find an infinite family of $M^{*}$-groups. Consequently the bound $12(g-1)$ is attained for infinitely many values of the genus $g$.

In the final section of the paper we show how to obtain other infinite families for which the bound $12(g-1)$ is achieved, without use of results about the modular group. To obtain an infinite family we only need a single Klein surface with boundary that has $12(g-1)$ automorphisms. Some of the infinite families we get in this manner consist of orientable surfaces; others consist of non-orientable surfaces.

1. Compact Klein surfaces and NEC groups. Let $X$ be a compact Klein surface, and let $E$ be the field of all meromorphic functions on $X . E$ is an algebraic function field in one variable over $\mathbf{R}[\mathbf{1}, \mathrm{p} .102]$ and as such has an algebraic genus $g$. This is the non-negative integer that makes the algebraic version of the Riemann-Roch Theorem work [2, p. 22]. We will refer to $g$ simply as the genus of the compact Klein surface $X$. In case $X$ is a Riemann surface, $g$ is equal to the topological genus of $X$.

Now let $\left(X_{c}, \pi, \sigma\right)$ be the complex double of $X$ [1, pp. 37-41], that is, $X_{c}$ is a compact Riemann surface, $\pi: X_{c} \rightarrow X$ is an unramified 2-sheeted covering of $X$, and $\sigma$ is the unique antianalytic involution of $X_{c}$ such that $\pi=\pi \circ \sigma$. If $F$ is the field of meromorphic functions on $X_{c}$, then $F=E(i)$ and by a well-known classical result [2, p. 99], the genus of $X$ is equal to the genus of $X_{c}$.

If $X$ and $Y$ are Klein surfaces, then a morphism [1, p. 17] is a continuous map $f: X \rightarrow Y$, with $f(\partial X) \subset \partial Y$ and the following local behavior. For every point $p \in X$ there exist dianalytic charts $(U, z)$ and $(V, w)$ at $p$ and $f(p)$ respectively such that $f(U) \subset V$ and $\left.f\right|_{U}=w^{-1} \circ \varphi \circ F \circ z$, where $F$ is an analytic function on $z(U)$ and $\varphi$ is the folding map, $\varphi(x+y i)=x+|y| i$.

Glasgow Math. J. 18 (1977) 1-10.

A 
To study automorphism groups of Klein surfaces with boundary, we use the theory of non-euclidean crystallographic (NEC) groups, as developed by Wilkie [11] and Macbeath [7]. Singerman [10] used NEC groups to obtain results about automorphism groups of nonorientable Klein surfaces without boundary.

Let $H=\{z \in \mathbf{C} \mid \operatorname{Im}(z)>0\}$ denote the open upper half-plane and $\Omega$ the group of automorphisms of $H$. The elements of $\Omega$ are transformations of one of two types:

$$
\begin{aligned}
& T(z)=\frac{a z+b}{c z+d}, \quad a d-b c=1, \quad a, b, c, d \quad \text { real; } \\
& T(z)=\frac{a \bar{z}+b}{c \bar{z}+d}, \quad a d-b c=-1, \quad a, b, c, d \quad \text { real. }
\end{aligned}
$$

Those of type (1.1) preserve orientation and form a subgroup $\Omega^{+}$of index 2 in $\Omega$. The only transformations of type (1.2) that have fixed points in $H$ are reflections (in case $a+d=0$ ). Reflections are of order two and have a circle of fixed points in $H$.

An NEC group is a subgroup of $\Omega$ that acts discontinuously on $H$. An NEC group contained in $\Omega^{+}$is called a Fuchsian group. If $\Gamma$ is an NEC group containing orientationreversing elements, then $\Gamma$ has a subgroup $\Gamma^{+}=\Gamma \cap \Omega^{+}$of index two.

Let $\Gamma$ be an NEC group such that the quotient space $H / \Gamma$ is compact. A fundamental region for $\Gamma$ is defined in the same way as for Fuchsian groups. We will denote the noneuclidean area of a fundamental region $F$ by $\mu(F)$. Many of the basic results about fundamental regions for Fuchsian groups continue to hold for fundamental regions for NEC groups. In particular, if $\Gamma^{\prime}$ is a subgroup of finite index in $\Gamma$ and $F, F^{\prime}$ are fundamental regions for $\Gamma, \Gamma^{\prime}$ respectively, then

$$
\left[\Gamma: \Gamma^{\prime}\right]=\mu\left(F^{\prime}\right) / \mu(F)
$$

An NEC group $\Gamma$ will be called a surface group if the quotient space $X=H / \Gamma$ is compact and the quotient map $p: H \rightarrow X$ is unramified. $\Gamma$ will be called a bordered surface group if further $\partial X \neq \varnothing$. Let $x \in H$. It is easy to see that $p(x) \in \partial X$ if and only if there exists a reflection $r \in \Gamma$ such that $r(x)=x$. Thus bordered surface groups contain reflections, but no other elements of finite order.

Now we recall two important results about the relationship between Klein surfaces and NEC groups.

THEOREM A. Let $\Gamma$ be an NEC group. Then the quotient space $H / \Gamma$ has a unique dianalytic structure such that the quotient map $p: H \rightarrow H / \Gamma$ is a morphism of Klein surfaces.

Proof. Since $\Gamma$ acts discontinuously on $H$, this follows immediately from a result of Alling and Greenleaf [1, p. 52].

THEOREM B. Let $X$ be a compact Klein surface with boundary of genus $g \geqq 2$. Then $X$ can be represented in the form $H / \Gamma$, where $\Gamma$ is a bordered surface group. If $F$ is a fundamental region for $\Gamma$, then

$$
\mu(F)=2 \pi(g-1)
$$


For a proof, see [9]. A good reference for the corresponding result about Riemann surfaces is $[6, p .66]$.

2. Induced mappings on the quotient space. This section was inspired by the treatment of the Riemann surface case in [6, pp. 59-63]. Also see [5].

For any Klein surface $Y$, let Aut $(Y)$ denote the group of automorphisms of $Y$. If $Y$ is orientable, let $\mathrm{Aut}^{+}(Y)$ denote the subgroup of orientation-preserving automorphisms.

Let $\Gamma$ be a bordered surface group. Let $X=H / \Gamma$ be the quotient space, $p: H \rightarrow X$ the quotient map. $X$ is a compact Klein surface with boundary, by Theorem $\mathrm{A}$.

A transformation $f \in \Omega$ is said to be $\Gamma$-admissible in case the following condition holds:

$$
p(x)=p(y) \text { if and only if } p(f(x))=p(f(y)) .
$$

If $f$ is $\Gamma$-admissible, then we can define a $\operatorname{map} f^{*}: X \rightarrow X$ by

$$
f^{*}(p(x))=p(f(x))
$$

It is easy to check that $f^{*}$ is an automorphism of $X$.

Let $A(H, \Gamma)$ denote the group of $\Gamma$-admissible automorphisms of $H$. Clearly $\Gamma \subset A(H, \Gamma)$. Let $N(\Gamma)$ denote the normalizer of $\Gamma$ in $\Omega$. It is not hard to see that $N(\Gamma) \subset A(H, \Gamma)$ (see $[6$, p. 60]).

Now the quotient space $H / \Gamma^{+}$is a compact Riemann surface. Let $q: H \rightarrow H / \Gamma^{+}$be the quotient map. Then the group of order two $C_{2}=\Gamma / \Gamma^{+}$acts on $H / \Gamma^{+}$to exhibit $H / \Gamma^{+}$ as an unramified double cover of $X=H / \Gamma$. If $\pi: H / \Gamma^{+} \rightarrow H / \Gamma$ denotes the quotient map, the following diagram commutes.

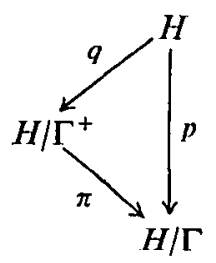

There is one point in the fiber $\pi^{-1}(x)$ if $x \in \partial X$; otherwise there are two. Let $f \in \Gamma-\Gamma^{+}$. Since $f \in N\left(\Gamma^{+}\right) \subset A\left(H, \Gamma^{+}\right)$, we can define an automorphism $\sigma$ of $H / \Gamma^{+}$by

$$
\sigma(q(x))=q(f(x)) \text {. }
$$

$\sigma$ is an orientation-reversing automorphism of order 2 and $\pi \circ \sigma=\pi$. Thus we see that $\left(H / \Gamma^{+}, \pi, \sigma\right)$ is the complex double of $X=H / \Gamma$. Now let $X_{c}$ denote $H / \Gamma^{+}$. We will use the complex double to establish the algebraic relationship between $\Gamma$ and $A(H, \Gamma)$ within $\Omega$. We have a group homomorphism $p^{*}: A(H, \Gamma) \rightarrow \operatorname{Aut}(X)$ defined by $p^{*}(f)=f^{*}$. We have a similar homomorphism $q^{*}: A\left(H, \Gamma^{+}\right) \rightarrow \operatorname{Aut}\left(X_{c}\right)$.

Proposition 1. $\Gamma=$ kernel $p^{*}$ and $A(H, \Gamma)=N(\Gamma)$.

Proof. Clearly $\Gamma \subset \operatorname{kernel} p^{*}$. Let $f \in \operatorname{kernel} p^{*}$ such that $f$ is analytic. Then $p=p \circ f$, i.e. $\pi \circ q=\pi \circ q \circ f$. Since $\left(X_{c}, \pi, \sigma\right)$ is the complex double of $X$, for each $x \in H$ either 
$q(x)=q \circ f(x)$ or $\sigma \circ q(x)=q \circ f(x)$. Suppose there exists a point $y \in H$ such that $q(y) \neq q \circ f(y)$. Then we can find an open set $U$ about $y$ such that $\sigma \circ q=q \circ f$ on $U$. Hence $f$ is antianalytic on $U$, a contradiction. Therefore $q=q \circ f$, and $f \in \operatorname{kernel} q^{*}$. Since $f$ is analytic, we have $f \in \Gamma^{+}[6$, p. 60].

If $h \in$ kernel $p^{*}$ and $h$ is antianalytic, then choose $k \in \Gamma-\Gamma^{+} . k \circ h$ is analytic and $k \circ h \in \operatorname{kernel} p^{*}$. Hence $k \circ h \in \Gamma^{+}$and $h \in \Gamma$. Therefore $\Gamma=\operatorname{kernel} p^{*}$.

Now $\Gamma$ is normal in $A(H, \Gamma)$. Thus $A(H, \Gamma) \subset N(\Gamma)$. We have already noted that $N(\Gamma) \subset A(H, \Gamma)$.

Proposition 2. $p^{*}: A(H, \Gamma) \rightarrow \operatorname{Aut}(X)$ is surjective.

Proof. Let $f \in \operatorname{Aut}(X)$. There exists a unique map $\tilde{f} \in \operatorname{Aut}^{+}\left(X_{c}\right)$ such that the following diagram commutes.

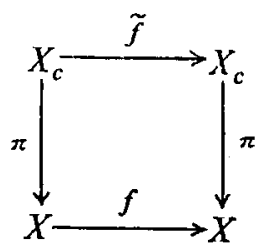

Moreover, $\sigma \circ \tilde{f} \circ \sigma=\tilde{f}$ and $\tilde{f}=\tilde{h}$ implies that $f=h[\mathbf{1}$, p. 79].

There is an analytic map $\theta \in A\left(H, \Gamma^{+}\right)$such that $q^{*}(\theta)=\tilde{f} \in \operatorname{Aut}^{+}\left(X_{c}\right)$ [6, p. 63]. Now $f \circ q=q \circ \theta$. It is easy to check that $\theta$ is $\Gamma$-admissible, so that we have an automorphism $p^{*}(\theta)=\theta^{*} \in \operatorname{Aut}(X)$. As before there is a unique map $\tilde{\theta}^{*} \in \operatorname{Aut}^{+}\left(X_{c}\right)$ such that $\pi \circ \tilde{\theta}^{*}=\theta^{*} \circ \pi$. But if $q(x) \in X_{c}, \pi \circ f(q(x))=\pi \circ q \circ \theta(x)=p \circ \theta(x)=\theta^{*} \circ p(x)=\theta^{*} \circ \pi(q(x))$. Hence $\tilde{f}=\tilde{\theta}^{*}$ and $f=\theta^{*}$, that is, $p^{*}(\theta)=f$. Thus $p^{*}$ is surjective.

Propositions 1 and 2 have the following important consequence.

PROPOSITION 3. Let $\Gamma$ be a bordered surface group. $G$ is a group of automorphisms of the Klein surface $H / \Gamma$ if and only if $G \cong \Delta / \Gamma$, where $\Delta$ is an $N E C$ group such that $\Gamma \subset \Delta \subset N(\Gamma)$.

The corresponding result about Riemann surfaces is similar and well known.

3. Large groups of automorphisms. The closed disc $D$ is a compact Klein surface of genus $0 ; D$ has (up to isomorphism) a unique dianalytic structure $[1, \mathrm{p} .60]$.

Let $G$ be a group of automorphisms of a compact Klein surface $X$ of genus $g \geqq 2$ with non-empty boundary. Then the quotient space $\Phi=X / G$ has a unique dianalytic structure such that the quotient map $\pi: X \rightarrow \Phi$ is a morphism of Klein surfaces. Moreover, $\pi$ is a ramified $r$-sheeted covering of $\Phi$, where $r=|G|$ (see [8] and [1, p. 52]).

Using a form of the Hurwitz ramification formula, we showed that $|G| \leqq 12(g-1)$. Also, $|G|=12(g-1)$ if and only if the quotient space $\Phi$ is the disc $D$, and the quotient map $\pi: X \rightarrow D$ is ramified above precisely 4 points of the boundary $\partial D$, the ramification indices $k_{i}$ in the fibers above these 4 points being $k_{1}=k_{2}=k_{3}=2, k_{4}=3$ [8].

Let $F$ be a four-sided non-euclidean polygon with angles $\frac{1}{2} \pi, \frac{1}{2} \pi, \frac{1}{2} \pi$, and $\frac{1}{3} \pi$. Let $A$ be the NEC group generated by the reflections $R_{1}, R_{2}, R_{3}, R_{4}$ in the sides of the polygon $F$. 
Then $A$ has the following presentation [3, $\mathrm{p} 55]$ :

$$
\left.\begin{array}{l}
\text { generators } R_{1}, R_{2}, R_{3}, R_{4}, \\
\text { and relations } \\
\qquad\left(R_{1}\right)^{2}=\left(R_{2}\right)^{2}=\left(R_{3}\right)^{2}=\left(R_{4}\right)^{2}=\left(R_{1} R_{2}\right)^{2}=\left(R_{2} R_{3}\right)^{2}=\left(R_{3} R_{4}\right)^{2}=\left(R_{4} R_{1}\right)^{3}=1 .
\end{array}\right\}
$$

The polygon $F$ is a fundamental region for the group $A$, of course, and by the Gauss-Bonnet Theorem [6, p. 21],

$$
\mu(F)=2 \pi-\frac{1}{2} \pi-\frac{1}{2} \pi-\frac{1}{2} \pi-\frac{1}{3} \pi=\frac{1}{6} \pi .
$$

Now suppose that a bordered surface group $\Gamma$ is a normal subgroup of finite index in $A$. Then $A / \Gamma$ is a group of automorphisms of the compact Klein surface $Y=H / \Gamma$. If $Y$ is of genus $g$, then

$$
|A / \Gamma|=[A: \Gamma]=\frac{2 \pi(g-1)}{\pi / 6}=12(g-1) .
$$

Thus we obtain a large automorphism group whenever we find a finite group $G$ and a homomorphism $\varphi: A \rightarrow G$ onto $G$ such that kernel $\varphi$ is a bordered surface group.

Before proceeding we need a definition.

Definttion. A finite group $G$ will be called an $M^{*}$-group in case $G$ is generated by three distinct elements $t, u, v$ of order two which satisfy the relations

$$
(t u)^{2}=(t v)^{3}=1 .
$$

Now we are ready to prove one of our main results.

THEOREM 1. G is a group of $12(g-1)$ automorphisms of a compact Klein surface of genus $g \geqq 2$ with non-empty boundary if and only if $G$ is an $M^{*}$-group.

Proof. Sufficiency. Suppose $G$ is an $M^{*}$-group with generators $t, u, v$ of order two that satisfy the relations (3). Define a homomorphism $\varphi: A \rightarrow G$ onto $G$ by $\varphi\left(R_{1}\right)=t, \varphi\left(R_{2}\right)=u$, $\varphi\left(R_{3}\right)=1, \varphi\left(R_{4}\right)=v$. Let $\Gamma=\operatorname{kernel} \varphi$. Then $R_{3} \in \Gamma$, and using a result of Macbeath [7, p. 1198], it is easy to see that any element of finite order in $\Gamma$ is conjugate to $R_{3}$ and thus is a reflection. Therefore $\Gamma$ is a bordered surface group. Now $G$ is a group of automorphisms of the Klein surface $Y=H / \Gamma$, and if $Y$ is of genus $g,|G|=12(g-1)$.

Necessity. Suppose $G$ is a group of $12(g-1)$ automorphisms of a compact Klein surface $X$ of genus $g \geqq 2$ with non-empty boundary. By Theorem $\mathrm{B}$, we can represent $X$ in the form $X=H / \Gamma$, where $\Gamma$ is a bordered surface group. Let $p: H \rightarrow X$ be the quotient map. Since $|G|=12(g-1)$ the quotient space $X / G$ is the disc $D$ and the quotient map $\pi: X \rightarrow D$ is ramified above exactly 4 points of $\partial D$, the ramification indices being $2,2,2,3$.

By Proposition 3, there exists an NEC group $B$, where $\Gamma \subset B \subset N(\Gamma)$, and a homomorphism $\varphi: B \rightarrow G$ onto $G$ such that kernel $\varphi=\Gamma$. Let $Y=H / B$ and let $q: H \rightarrow Y$ be the quotient map. It is easy to check that the map $f: Y \rightarrow D$ defined by $f(q(x))=\pi(p(x))$ is a 
dianalytic isomorphism. Thus the quotient space $H / B$ is the disc $D$, and the following diagram commutes.

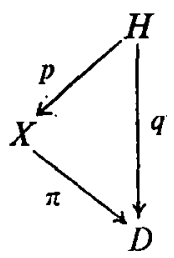

The map $p$ is unramified. Therefore $q$ is ramified above precisely the same points of $D$ that $\pi$ is, and the ramification indices above these points must also be the same. Then it follows from Wilkie's results [11, p. 96] that, after a simple elimination of redundant generators, the group $B$ has the presentation (2).

Now $\Gamma$ is a bordered surface group, so that $\Gamma$ contains a reflection. Since $\Gamma$ is normal in $B$, it follows that one of the $R_{i}$ is in $\Gamma$ [7, p. 1198]. Suppose $R_{1} \in \Gamma=\operatorname{kernel} \varphi$. Since $R_{4}^{2}=\left(R_{4} R_{1}\right)^{3}=1, R_{4} \in \Gamma$ as well. Then $R_{4} R_{1}$ is an analytic element of finite order in the surface group $\Gamma$, a contradiction. Hence $R_{1} \notin \Gamma$. Similarly $R_{4} \notin \Gamma$.

Suppose $R_{3} \in \Gamma$. Let $t=\varphi\left(R_{1}\right), u=\varphi\left(R_{2}\right)$, and $v=\varphi\left(R_{4}\right)$. Then $t, u, v$ generate $G$ and satisfy the relations (3). Hence $G$ is an $M^{*}$-group.

If $R_{2} \in \Gamma$, then let $t=\varphi\left(R_{4}\right), u=\varphi\left(R_{3}\right)$, and $v=\varphi\left(R_{1}\right)$. Again we see that $G$ is an $M^{*}$-group. This concludes the proof.

The proof indicates the relationship between [8] and the work of Wilkie [11].

4. $\mathbf{M}^{*}$-groups. Let $\Gamma$ be the extended modular group, the subgroup of $\Omega$ consisting of all transformations of types (1.1) and (1.2) where $a, b, c, d$ are integers. Then $\Gamma^{+}$is the usual modular group. The group $\Gamma$ is generated by the transformations $T, U, V$ where $T(z)=1 / \bar{z}, U(z)=-\bar{z}$, and $V(z)=-\bar{z} /(\bar{z}+1)$. They satisfy the relations

$$
T^{2}=U^{2}=V^{2}=(T U)^{2}=(T V)^{3}=1,
$$

and these relations actually define $\Gamma\left[3\right.$, pp. 85-86]. Immediately we see that $M^{*}$-groups are finite quotient groups of $\Gamma$. On the other hand, suppose $K$ is any normal subgroup of $\Gamma$ of finite index larger than 6 . It can be checked that $T, U, V, T U$, and $T V$ are not in $K$. Therefore $\Gamma / K$ is an $M^{*}$-group.

For each integer $q \geqq 2$, let $\Gamma_{q}$ be the principal congruence subgroup of level $q$, consisting of all transformations $(1.1)$ of $\Gamma^{+}$such that $a \equiv d \equiv \pm 1(\bmod q)$, and $b \equiv c \equiv 0(\bmod q)$. Let $\mu=\left[\Gamma^{+}: \Gamma_{q}\right]$ be the index of $\Gamma_{q}$ in $\Gamma^{+}$. If $q=2$, then $\mu=6$. If $q>2$, then

$$
\mu=\frac{1}{2} q^{3} \prod_{p \mid q}\left(1-\frac{1}{p^{2}}\right),
$$

where the product is over the prime divisors of $q[4, \mathrm{pp} .8-10]$. Now note that $\Gamma_{q}$ is normal in the big group $\Gamma$. $\left[\Gamma: \Gamma_{q}\right]=2 \mu \geqq 12$. Thus we have the following important result.

THEOREM 2. For each integer $q \geqq 2, \Gamma / \Gamma_{q}$ is an $M^{*}$-group.

COROLLARY. There are infinitely many values of the genus $g$ for which there is a compact Klein surface with boundary with $12(g-1)$ automorphisms. 
It is possible to find one topological type of Klein surface with boundary on which $\Gamma / \Gamma_{q}$ acts. Consider the quotient space $H / \Gamma_{q}$. For more details on the following, see the treatment in [4, pp. 6-16]. Let $M$ denote the compactification of $H / \Gamma_{q} . M$ is a compact Riemann surface of genus $h$, where $h=0$ if $q=2$, and

$$
h=1+\frac{q^{2}(q-6)}{24} \prod_{p \mid q}\left(1-\frac{1}{p^{2}}\right) \text { if } q>2 .
$$

The quotient group $\Gamma / \Gamma_{q}$ acts as a group of automorphisms of $M$. Let $\lambda=\mu / q$. There are exactly $\lambda$ points of the quotient space $M$ that are images of points equivalent under $\Gamma^{+}$(or under $\Gamma$ ) to $i \infty$. These $\lambda$ points form an orbit of the quotient group $\Gamma / \Gamma_{q}$. Now remove an open disc centered about each of these $\lambda$ points, and let $X$ denote the resulting surface. $X$ inherits an analytic structure from $M$, and $\Gamma / \Gamma_{q}$ acts as a group of automorphisms of $X$. Topologically $X$ is a sphere with $h$ handles and $\lambda$ holes. Then $X$ is of genus $g=2 h+\lambda-1$ [1]. We need to calculate the order of $\Gamma / \Gamma_{q}$ in terms of the genus $g$. From [4, p. 14] we have the following relation.

$$
12(h-1)=\mu(1-6 / q)=\mu-6 \lambda .
$$

Therefore $\left|\Gamma / \Gamma_{q}\right|=2 \mu=2[12(h-1)+6 \lambda]=12[2(h-1)+\lambda]=12[(2 h+\lambda-1)-1]=12(g-1)$. Thus $\Gamma / \Gamma_{q}$ acts as an automorphism group of $X$ of maximum possible order.

The values of $2 \mu, g, h, \lambda$ are tabulated below for some small values of $q$.

\begin{tabular}{|c|rrrr|r|rrrr|}
\hline$q$ & $2 \mu$ & $g$ & $h$ & $\lambda$ & $q$ & $2 \mu$ & $g$ & $h$ & $\lambda$ \\
\hline 2 & 12 & 2 & 0 & 3 & 7 & 336 & 29 & 3 & 24 \\
3 & 24 & 3 & 0 & 4 & 8 & 384 & 33 & 5 & 24 \\
4 & 48 & 5 & 0 & 6 & 9 & 648 & 55 & 10 & 36 \\
5 & 120 & 11 & 0 & 12 & 10 & 720 & 61 & 13 & 36 \\
6 & 144 & 13 & 1 & 12 & 11 & 1320 & 111 & 26 & 60 \\
\hline
\end{tabular}

Before proceeding, we give a table of $M^{*}$-groups of low order that we have found. These groups are quite familiar, and it is easy enough to check that they are $M^{*}$-groups. $S_{n}$ denotes the symmetric group on $n$ letters, $A_{n}$ the alternating group on $n$ letters, and $C_{n}$ the cyclic group of order $n$.

$M^{*}$-groups of low order

\begin{tabular}{|l|r|r|}
\hline \multicolumn{1}{|c|}{ Group } & Order & Genus \\
\hline$C_{2} \times S_{3}$ & 12 & 2 \\
$S_{4}$ & 24 & 3 \\
$S_{3} \times S_{3}$ & 36 & 4 \\
$C_{2} \times S_{4}$ & 48 & 5 \\
$A_{3}$ & 60 & 6 \\
$C_{2} \times A_{3}$ & 120 & 11 \\
$S_{3} \times S_{4}$ & 144 & 13 \\
$C_{2} \times C_{2} \times A_{5}$ & 240 & 21 \\
\hline
\end{tabular}


5. Infinite families. In this section we exhibit other infinite families of surfaces for which the bound $12(g-1)$ is attained. The technique used is essentially the same as that employed in [5] and [10].

Suppose $\Gamma$ is a bordered surface group such that the quotient space $H / \Gamma$ is of genus $g$ and has $12(g-1)$ automorphisms. Then, as in the proof of Theorem 1 , there exists an NEC group $B$ with presentation (2), such that $\Gamma$ is a normal subgroup of $B$ and $[B: \Gamma]=12(g-1)$.

The commutator subgroup $\Gamma^{\prime}$ is a characteristic subgroup of $\Gamma$. The subgroup $\Gamma^{m}$ of $\Gamma$ generated by the $m^{\text {th }}$ powers of elements of $\Gamma$ is also a characteristic subgroup. $\Gamma^{m}$ contains orientation-reversing elements if and only if $m$ is odd.

For odd $m$, let $\Delta_{m}=\Gamma^{\prime} \Gamma^{m}$. $\Delta_{m}$ is a characteristic subgroup of $\Gamma$. $\Gamma$ is finitely generated by Wilkie's results [11]. The quotient group $\Gamma / \Delta_{m}$ is a finitely generated abelian group in which every element has finite order. Thus $\Gamma / \Delta_{m}$ is a finite abelian group, so that $\Delta_{m}$ is of finite index in $\Gamma$. Hence $\Delta_{m}$ is a surface group. But $\Gamma$ contains reflections, and since $m$ is odd, so does $\Delta_{m}$. Thus $\Delta_{m}$ is a bordered surface group. Since $\Delta_{m}$ is characteristic in $\Gamma, \Delta_{m}$ is normal in $B$. Now, as we have seen, $H / \Delta_{m}$ is a compact Klein surface with boundary of genus $g^{\prime}$ that has $12\left(g^{\prime}-1\right)$ automorphisms. When the topological type of $H / \Gamma$ is known, we can calculate the genus of each Klein surface $H / \Delta_{m}$. For example, we have the following.

LEMma 1. Suppose $H / \Gamma$ topologically is a sphere with $k$ holes. Then the genus $g^{\prime}$ of $H / \Delta_{m}$ is

$$
g^{\prime}=(k-2) m^{k-1}+1
$$

Further, each surface $H / \Delta_{m}$ is orientable.

Proof. $\Gamma$ has the following canonical presentation $[11$, p. 96]:

$$
\begin{aligned}
& \text { generators } c_{1}, \ldots, c_{k}, e_{1}, \ldots, e_{k}, \\
& \text { and relations } c_{i}^{2}=e_{i} c_{i} e_{i}^{-1} c_{i}=1 \quad(i=1, \ldots, k), e_{1} e_{2} \ldots e_{k}=1 .
\end{aligned}
$$

Now $c_{i}^{m}=c_{i} \in \Delta_{m}$ for each $i$. Therefore $\Gamma / \Delta_{m}$ has the presentation:

$$
\begin{aligned}
& \text { generators } \bar{e}_{1}, \ldots, \bar{e}_{k}, \\
& \text { and relations } m \bar{e}_{i}=0 \quad(i=1, \ldots, k), \quad \bar{e}_{1}+\bar{e}_{2}+\ldots+\bar{e}_{k}=0 .
\end{aligned}
$$

Clearly $\Gamma / \Delta_{m} \cong\left(C_{m}\right)^{k-1}$, so that $\left[\Gamma: \Delta_{m}\right]=m^{k-1}$. The genus of the Klein surface $H / \Gamma$ is $(k-1)$. Both $\Gamma$ and $\Delta_{m}$ are bordered surface groups. Therefore

$$
m^{k-1}=\left[\Gamma: \Delta_{m}\right]=\frac{2 \pi\left(g^{\prime}-1\right)}{2 \pi[(k-1)-1]}
$$

Then $g^{\prime}=(k-2) m^{k-1}+1$.

Let $X=H / \Gamma$ and $Y=H / \Delta_{m}$, and let $p: H \rightarrow X$ and $q: H \rightarrow Y$ be the quotient maps. Then $G=\Gamma / \Delta_{m}$ is a group of automorphisms of $Y$. As in the proof of Theorem 1, the quotient space $Y / G$ is dianalytically isomorphic to $X$. If $\pi: Y \rightarrow X$ denotes the quotient map, then $p=\pi \circ q . \pi$ is an unramified $m^{k-1}$-sheeted covering of $X$, since $|G|=m^{k-1}$ [8]. Suppose $\pi(q(x))=p(x) \in \partial X$. Then there is a reflection $r \in \Gamma$ with $r(x)=x$. But $r \in \Delta_{m}$ as well, since $m$. 
is odd, so that $q(x) \in \partial Y$. Thus $\pi^{-1}(\partial X)=\partial Y$, that is, $Y$ is not folded along $\partial X$. Hence we can lift the orientation of $X$ to define an orientation of $Y$.

THEOREM 3. There is a compact orientable Klein surface of genus $g$ with non-empty boundary that has $12(g-1)$ automorphisms for each of the following values of $g$ :

$$
g=m^{2}+1, \quad g=2 m^{3}+1, \quad g=4 m^{5}+1, \quad g=10 m^{11}+1,
$$

where $m$ is any positive odd integer.

Proof. Lemma 1 applies to 4 examples of the previous section (see the first table with $q=2,3,4,5)$.

To find infinite families of non-orientable surfaces, we first need examples of nonorientable surfaces with $12(g-1)$ automorphisms.

EXAMPLE. Let $X$ be a sphere with 12 holes, with the holes centered around the vertices of an inscribed regular icosahedron. $X$ is an orientable Klein surface of genus $11 . X$ has a group of automorphisms isomorphic to the complete symmetry group (including reflections) of the regular icosahedron, which is $C_{2} \times A_{5}$. Let $\tau: X \rightarrow X$ be the antipodal map. The quotient space $W=X / \tau$ is a real projective plane with 6 holes, a non-orientable Klein surface of genus 6. $W$ has an automorphism group isomorphic to $A_{5}$. Thus $W$ has $60=12(6-1)$ automorphisms.

A similar example in [8] gives a non-orientable Klein surface of genus 3, topologically a real projective plane with 3 holes, that has 24 automorphisms.

LEMMA 2. Suppose $H / \Gamma$ topologically is a real projective plane with $k$ holes. Then the genus $g^{\prime}$ of $H / \Delta_{m}$ is

$$
g^{\prime}=(k-1) m^{k}+1 \text {. }
$$

Further, each surface $\mathrm{H} / \Delta_{m}$ is non-orientable.

Proof. $\Gamma$ has the following canonical presentation [11, p. 101]:

generators $c_{1}, \ldots, c_{k}, e_{1}, \ldots, e_{k}, d$,

and relations $c_{i}^{2}=1 \quad(i=1, \ldots, k), \quad e_{i} c_{i} e_{i}^{-1} c_{i}=1 \quad(i=1, \ldots, k), \quad d^{2} e_{1} \ldots e_{k}=1$.

Then $\Gamma / \Delta_{m}$ has the following presentation:

generators $\bar{e}_{1}, \ldots, \bar{e}_{k}, \quad \bar{d}$,

and relations $2 \bar{d}+\bar{e}_{1}+\ldots+\bar{e}_{k}=0, \quad m \bar{d}=0, \quad m \vec{e}_{i}=0 \quad(i=1, \ldots, k)$.

Thus $\Gamma / \Delta_{m} \cong\left(C_{m}\right)^{k}$ and $\left[\Gamma: \Delta_{m}\right]=m^{k}$. The genus of the Klein surface $H / \Gamma$ is $k$. Therefore

and $g^{\prime}=(k-1) m^{k}+1$.

$$
m^{k}=\left[\Gamma: \Delta_{m}\right]=\frac{2 \pi\left(g^{\prime}-1\right)}{2 \pi(k-1)}
$$

Now, as in the proof of Lemma 1, let $X=H / \Gamma, Y=H / \Delta_{m}$, and $G=\Gamma / \Delta_{m}$. The quotient space $Y / G$ is the Klein surface $X$. If $\pi: Y \rightarrow X$ denotes the quotient map, then $\pi$ is an unramified $m^{k}$-sheeted covering of $X$ and $\pi^{-1}(\partial X)=\partial Y$. Suppose $Y$ is orientable. Then let 
$\left(X_{0}, v, \tau\right)$ be the orienting double of $X[1, \mathrm{pp} .42-43]$, that is, $X_{o}$ is a compact orientable Klein surface with boundary, $v: X_{o} \rightarrow X$ is an unramified 2-sheeted covering of $X$, and $\tau$ is the unique antianalytic involution of $X_{o}$ such that $v_{\circ} \tau=v$. Since $\pi^{-1}(\partial X)=\partial Y$, there exists a unique analytic map $f: Y \rightarrow X_{o}$ such that the following diagram commutes [1, p. 42].

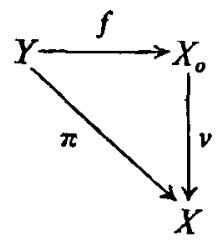

Then $f$ is an $r$-sheeted covering of $X_{o}$ for some integer $r$, and $2 r=m^{k}$. But $m^{k}$ is odd, a contradiction. Therefore $Y$ is non-orientable.

THEOREM 4. There is a compact non-orientable Klein surface of genus $g$ with non-empty boundary that has $12(g-1)$ automorphisms for each of the following values of $g$ :

$$
g=2 m^{3}+1, g=5 m^{6}+1,
$$

where $m$ is any odd positive integer.

We are working (with Newcomb Greenleaf) on other techniques for finding infinite families of surfaces for which the bound $12(g-1)$ is attained. A sequel is planned.

Thanks are due the referee for calling our attention to the relationship of the extended modular group to $M^{*}$-groups.

\section{REFERENCES}

1. N. L. Alling and N. Greenleaf, Foundations of the theory of Klein surfaces, Lecture Notes in Mathematics No. 219 (Springer-Verlag, 1971). Soc., 1951).

2. C. Chevalley, Introduction to the theory of algebraic functions of one variable (Amer. Math.

3. H. S. M. Coxeter and W. O. J. Moser, Generators and relations for discrete groups (SpringerVerlag, 1972).

4. R. C. Gunning, Lectures on modular forms (Princeton, 1962).

5. A. M. Macbeath, On a theorem of Hurwitz, Proc. Glasgow Math. Assoc. 5 (1961), 90-96.

6. A. M. Macbeath, Discontinuous groups and birational transformations, Proceedings of the Summer School, Queen's College, Dundee (1961).

7. A. M. Macbeath, The classification of non-euclidean plane crystallographic groups, Canad. J. Math. 19 (1967), 1192-1205.

8. C. L. May, Automorphisms of compact Klein surfaces with boundary, Pacific J. Math. 59 (1975), 199-210.

9. R. Preston, Projective structures and fundamental domains on compact Klein surfaces, Ph.D. Thesis, The University of Texas (1975).

10. D. Singerman, Automorphisms of non-orientable Riemann surfaces, Glasgow Math. J. 12 (1971), 50-59.

11. H. C. Wilkie, On non-euclidean crystallographic groups, Math. Z. 91 (1966), 87-102.

The University of TeXas at Austin

Present address: UNIVERSITY OF KeNTUCKY Austin, TeXas 78712, U.S.A.

LEXINGTON, KENTUCKY 40506, U.S.A. 